\title{
FUNDAMENTALS OF SOLUBILITY ENHANCEMENT TECHNIQUES: WHAT DO WE NEED TO CONSIDER?
}

\author{
Sunita Dahiya* \\ Pharmaceutical Sciences Department, School of Pharmacy, University of Puerto Rico, Medical Sciences \\ Campus, San Juan, PR 00931, Puerto Rico \\ *E-mail: sunita.dahiya@upr.edu \\ Tel.: +1 7872456385 .
}

Received: Jan 16, 2017 / Revised: Mar 10, 2017 / Accepted: Mar 11, 2017

The increasing number of poorly water soluble drugs requires innovative formulation approaches to reach a sufficiently high bioavailability after oral administration or intravenous route. There are a number of classical formulation approaches which are of limited success as demonstrated by the relatively low number of products on the market being based on such technologies. In the recent years, nanoparticle technology has emerged as a strategy to tackle such formulation problems associated with poorly water soluble drugs. The reduction of drug particle to the nano-scale increases dissolution velocity and saturation solubility, and this change of materials into the nanodimension dramatically change its physical properties, leading to improved in vivo drug performance. In the context of challenges associated with limited drug solubility, this review imparts some theoretical basis to address solubility as a key determinant to bioavailability and outlines basic pharmaceutical approaches to overcome poor solubility problem of drug candidates.

Key words: Solubility, Bioavailability, Particle size, Nanonization.

\section{INTRODUCTION}

Together with the permeability, the solubility behavior of a drug is a key determinant of its oral bioavailability. There have always been certain drugs for which solubility has presented a challenge to the development of a suitable formulation for oral administration. Examples such as griseofulvin, digoxin, phenytoin, sulphathiazole and chloramphenicol come immediately to mind. With the recent advent of high throughput screening of potential therapeutic agents, the number of poorly soluble drug candidates has risen sharply and the formulation of poorly soluble compounds for oral delivery now presents one of the most frequent and greatest challenges to formulation scientists in the pharmaceutical industry. Consideration of the modified equation (Noyes and Whitney, 1897) provides some hints as to how the dissolution rate of even very poorly soluble compounds might be improved to minimize the limitations to oral availability:

$\mathrm{dc} / \mathrm{dt}=\mathrm{AD}(\mathrm{Cs}-\mathrm{C}) / \mathrm{h}$

where $\mathrm{dc} / \mathrm{dt}$ is the rate of dissolution, $\mathrm{A}$ is the surface area available for dissolution, $D$ is the diffusion coefficient of the compound, Cs is the solubility of the compound in the dissolution medium, $\mathrm{C}$ is the concentration of drug in the medium at time $\mathrm{t}$ and $\mathrm{h}$ is the thickness of the diffusion boundary layer adjacent to the surface of the dissolving compound.

The main possibilities for improving dissolution according to this analysis are to increase the surface area available for dissolution by decreasing the particle size of the solid compound and/or by optimizing the wetting characteristics of the compound surface, to 
decrease the boundary layer thickness, to ensure sink conditions for dissolution and, last but definitely not least, to improve the apparent solubility of the drug under physiologically relevant conditions. Of these possibilities, changes in the hydrodynamics are difficult to invoke in vivo and the maintenance of sink conditions will depend on how permeable the gastrointestinal mucosa is to the compound as well as on the composition and volume of the lumenal fluids. Although some research efforts have been directed towards permeability enhancement using appropriate excipients, results to date have not been particularly encouraging. Administration of the drug in the fed state may be an option to improve the dissolution rate and also to increase the time available for dissolution; the likely magnitude of the food effect can be forecasted from dissolution tests in biorelevant media (Galia et al 1998). However, much the most attractive option for increasing the release rate is improvement of the solubility through formulation approaches.

Of the physical approaches, literature reports are available on the use of polymorphs (Henck et al 1997), the amorphous form of the drug (Hancock and Zografi, 1997) and complexation (Horter and Dressman, 2001; Loftsson and Brewster, 1996). Decreasing the particle size of the compound by milling the drug powder theoretically results in an increase in the available area for dissolution, but in some cases the micronized powder tends to agglomerate, thereby at least partly negating the milling procedure. The use of very fine powder in a dosage forms may also problematic because of handling difficulties and poor wettability. Presenting the compound as a molecular dispersion combines the benefits of a local increase in the solubility (within the solid solution) and maximizing the surface area of the compound that comes in contact with the dissolution medium as the carrier dissolves. Among chemical approaches, the salt formation is not feasible for neutral compounds and the synthesis of appropriate salt forms of drugs that are weakly acidic or weakly basic may often not be practical. Even when salts can be prepared and increased dissolution rate in gastrointestinal tract may not be achieved in many cases because of the conversion of salts into aggregates of their respective acid or base forms. The solubilization of the drugs in organic solvents or in aqueous media by the use of surfactants and co-solvents leads to liquid formulations that are usually undesirable from the view points of patients' acceptability and commercialization. Although particle size reduction is commonly to increase the dissolution rate, there is a practical limit to how much size reduction can be achieved by such commonly used methods such as conventional trituration and grinding, ball milling, fluid energy micronization, controlled precipitation by change of solvents or temperature, administration of liquid solutions from which, upon dilution with gastric fluids, the dissolved drug may precipitate in very fine particles, administration of water-soluble salts of poorly soluble compounds from which the parent neutral forms may precipitate in ultra fine forms in G.I. fluids.

\section{Poor solubility: A major challenge to drug delivery}

High hydrophobicity and intrinsically low water solubility are increasingly common characteristics of hits, leads, development candidates, and ultimately marketed drugs. Many hypotheses have been put forward as to why these trends have emerged, and the true explanation is clearly multifaceted. The application of combinatorial chemistry to generate large chemical libraries and the common application of high-throughput screening modalities, often in non-aqueous media (or mixed solvent media) have probably played a role. The desire for increased potency, coupled with the realization that receptor binding is mediated, at least in part, by hydrophobic interactions, further magnifies the likelihood that drug candidates will have limited aqueous solubility. Finally, the quest to explore unprecedented drug targets, some of which are associated with intracellular signaling pathways, lipid processing architecture, or highly lipophilic endogenous ligands, only amplifies the requirement for highly lipophilic, poorly watersoluble drug candidates to access and interact with the target. These drivers ultimately bias the identification of poorly water-soluble "hits" during early drug screens. Poor water solubility is a significant risk factor in low oral absorption because drug molecules must, in most cases, be in solution to be absorbed, and oral bioavailability is usually a required characteristic in a target product profile of an orally administered medicine. As such, medicinal chemistry strategies during lead optimization typically seek to modify physicochemical 
properties (including solubility) such that drug leads have more developable characteristics. Many decision gates, or idealized character panels, are used to identify and reject drug candidates with inappropriate developability properties and, subsequently, to synthetically modify structures to improve physicochemical characteristics.

Perhaps the best known of these is Chris Lipinski's "rule of 5", but there are many others. In all cases, however, at least moderate water solubility is usually a focus. Nonetheless, even with contemporary medicinal chemistry programs and increasingly sophisticated lead optimization strategies, it is apparent that for some targets, reducing lipophilicity and increasing water solubility will result in an unacceptable reduction in potency.

In spite of attempts to circumvent solubility problems, approximately $40 \%$ of currently marketed drugs and up to $75 \%$ of compounds currently under development have been suggested to be poorly water-soluble. Furthermore, the problems of low water solubility do not seem to be diminishing and may well be increasing.

Low water solubility therefore continues to be a challenge to successful drug development. Although low water solubility of drug candidates presents varied and significant challenges throughout drug discovery and development, the greatest concern is generally the risk of reduced and variable absorption after oral administration. The value at which limited solubility begins to impact absorption is difficult to state definitively since it is dependent on a number of other system variables, including drug permeation, dose, and the environment present within the gastrointestinal (GI) tract.

In the absence of supersaturation, the maximum concentration that can be attained at the surface of an absorptive membrane is equivalent to the equilibrium solubility $\left(\mathrm{C}_{\mathrm{s}}\right)$ of the drug, and therefore the maximum flux (per unit area) (F') is the product of solubility and permeability:

$F^{\prime}=P . C_{s}$

Appreciation of this relationship illustrates that knowledge of the solubility alone is insufficient to anticipate whether solubility will limit flux (or absorption) since flux is also a function of permeability. To some extent, therefore, low solubility can be offset by high permeability; similarly, if permeability is low, the requirements for solubility to generate appropriate flux increase. A well recognized approach applied in early drug discovery for estimating the required solubility and permeability needed to achieve good oral absorption is the concept of a maximum absorbable dose (MAD):

$\mathrm{MAD}=\mathrm{C}_{\mathrm{s}} \times \mathrm{k}_{\mathrm{abs}} \times \mathrm{SIWV} \times \mathrm{SITT}$

where $\mathrm{C}_{\mathrm{s}}$ is the solubility $(\mathrm{mg} / \mathrm{ml})$ at $\mathrm{pH} 6.5$ (representing the $\mathrm{pH}$ of the small intestine); kabs is the rate constant $\left(\mathrm{h}^{-1}\right)$ for intestinal absorption (which is related to the permeability); SIWV is the small intestinal water volume (in milliliters), which is typically assumed to be $250 \mathrm{ml}$ (the volume of fluid assumed to be present in the fasted GI tract after a glass of water has been drunk when taking medication orally); and SITT is the small intestinal transit time (min) of 270 $\min (4.5 \mathrm{~h})$.

Rearranging this relationship provides an expression for the necessary or target solubility for a given dose and $\mathrm{k}_{\mathrm{abs}}$ (or permeability) and provides an initial indication as to whether solubility is likely to limit oral absorption. This concept is shown graphically in Figure 1, the data from which are taken from a seminal review that shows the theoretical required solubility to provide good oral absorption for drugs with projected doses ranging from 0.1 to $10 \mathrm{mg} / \mathrm{kg}$ and permeabilities ranging from low to high.

At one end of the spectrum, highly potent drugs for which the dose is low and the membrane permeability is high have relatively low solubility requirements to achieve good oral absorption. At the other extreme, low-potency drugs for which the dose is high and the permeability is low need considerably higher solubility for good oral absorption (by several orders of magnitude in this example). This approach also indicates that where aqueous solubilities are, $50 \mathrm{mg} / \mathrm{ml}$, problems associated with low water solubility might be anticipated. It is clear, however, that the required solubility to support drug absorption must be evaluated in light of both the potency (or dose) and the permeability characteristics.

It is also clear that at stages during the development pathway; in particular, during preclinical toxicity testing, exposure at doses considerably in excess of the predicted clinical dose will be required, magnifying the need for solubility support. 


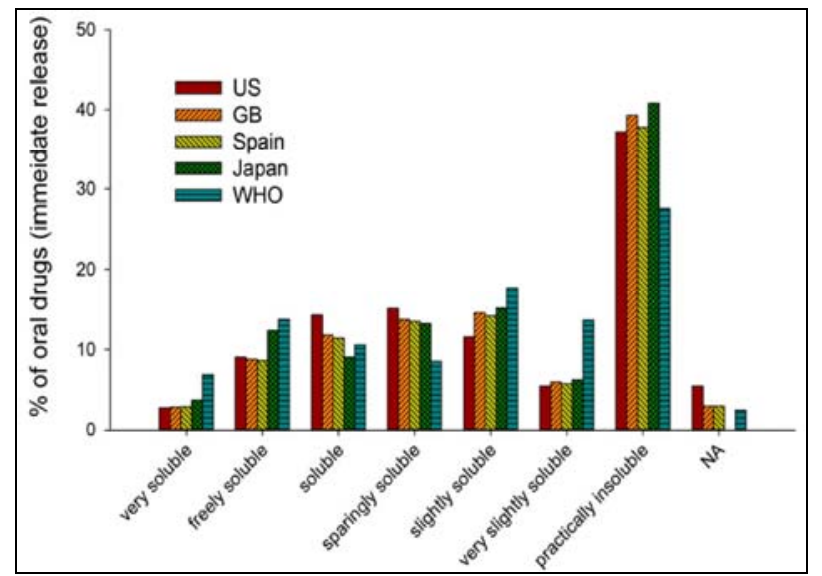

Fig. 1. A comparison of the distribution of solubilities for the top 200 oral drug products in the United States (US), Great Britain (GB), Spain, and Japan and from the World Health Organization (WHO) Essential Drug List. Very soluble drugs: over $1000 \mathrm{mg} / \mathrm{ml}$; freely soluble drugs: $100-1000 \mathrm{mg} / \mathrm{ml}$; soluble drugs: $33-100$ $\mathrm{mg} / \mathrm{ml}$; sparingly soluble drugs: $10-33 \mathrm{mg} / \mathrm{ml}$; slightly soluble drugs: $1-10 \mathrm{mg} / \mathrm{ml}$; very slightly soluble drugs: $0.1-1 \mathrm{mg} / \mathrm{ml}$; practically insoluble drugs: $0.1 \mathrm{mg} / \mathrm{ml}$. (Takagi et al 2006).

A significant application of the solubilitypermeability relationship to oral drug absorption is the Biopharmaceutics Classification System (BCS) (Figure 2) (Amidon et al 1995; Wu and Benet, 2005). The principles of the BCS are well described in literature (Yu et al 2002; Dahan et al 2009), but in brief, the BCS allows classification of drug molecules as a function of their solubility and permeability properties. Originally proposed to provide a scientific basis for biowaivers based on a correlation of in vitro drug dissolution and in vivo drug absorption, this classification system has proved to be a potential scientific tool showing much broader applicability across many areas of drug discovery and development. According to the BCS, class I molecules are those having both high solubility and high permeability (and therefore likely few problems with oral absorption); class II compounds are those that have low solubility and high permeability (where solubility is the primary limitation to absorption); class III compounds have high solubility but low permeability (where absorption is limited by membrane permeation and not solubility); and class IV compounds are those in which both poor solubility and poor permeability limit drug absorption. The focus of the current review is therefore BCS class II compounds, which often exhibit solubility- limited absorption. Class IV compounds are also relevant, although they have additional problems associated with low permeability.

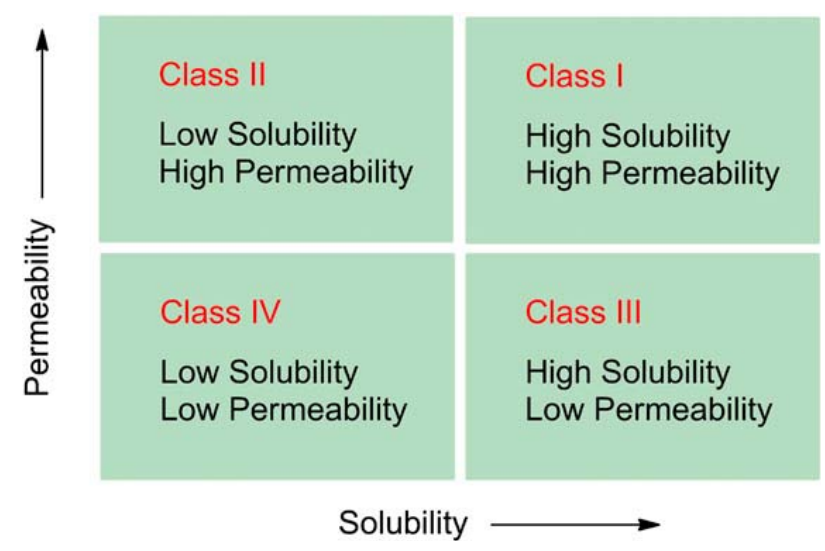

Fig. 2. Diagrammatic representation of the BCS, which classifies drugs according to their permeability and solubility properties.

Drug dose is also an important factor in the BCS because highly soluble drugs are defined as those in which the highest dose will dissolve in $250 \mathrm{ml}$ over the $\mathrm{pH}$ range of the GI tract (i.e. $\mathrm{pH}$ 1-6.8). As with the MAD, dose, solubility and volume calculations are not designed to be definitive but rather illustrate that low-dose compounds, such as digoxin, can have good absorption and bioavailability, even when GI solubility is low, whereas the absorption of highdose compounds, such as griseofulvin, is more often low, variable, and highly formulation dependent as a result of their solubility limitations. As per the BCS definition of high solubility, the highest strength dose must be soluble in $250 \mathrm{ml}$ of water at all $\mathrm{pH}$ values that might be encountered in the GI tract. Therefore, drugs may be classified as class II even though they have good solubility at one end of this $\mathrm{pH}$ range. For example, many weak acids have low solubility at $\mathrm{pH} 1$ and are strictly classified as BCS class II compounds but are quite soluble at intestinal $\mathrm{pH}(\mathrm{pH} \mathrm{6-7)}$ and in many cases do not exhibit solubility-limited absorption. A BCS class II designation does not always dictate that solubility will be a limitation to absorption; rather, compounds in this BCS class are more likely to be solubility limited than are those in class I. Indeed a more practical description of BCS class II may be drugs that do not have high solubility rather than those that have low solubility since the classification system specifically identifies compounds that fall into class I and all those with solubilities below this fall inherently into class II (or class IV if 
permeability is also low). This discussion illustrates that low solubility is a somewhat arbitrary concept when assessing the likelihood that solubility will limit drug absorption. Additional knowledge of the likely dose and membrane permeability is inevitably required to put a solubility value into an appropriate context.

\section{Approaches employed for solubility and dissolution enhancement}

Solubility improvement techniques can be categorized in to physical modification, chemical modifications of the drug substance, and other techniques.

\section{Physical modifications}

Particle size reduction like micronization and nanosuspension, modification of the crystal habit like polymorphs, amorphous form and cocrystallization, drug dispersion in carriers like eutectic mixtures, solid dispersions, solid solutions and cryogenic techniques.

\section{Chemical modifications}

Change of $\mathrm{pH}$, use of buffer, derivatization, complexation, and salt formation.

\section{Miscellaneous methods}

Supercritical fluid process, use of adjuvant like surfactant, solubilizers, cosolvency, hydrotrophy, and novel excipients.

The brief outline of approaches employed for dissolution enhancement of poorly soluble drugs is given below.

\section{Metastable polymorphs}

Polymorphism in crystalline solids is defined as materials with the same chemical composition, but different lattice structures and/or different molecular conformations. The vast majority of drugs can crystallize into several polymorphs. Each polymorph has a different energy, showing different physicochemical properties, such as melting point, density, solubility, and stability. Generally, the solubility of metastable polymorphs is kinetically higher than that of a thermodynamically more stable polymorph. The differences of the solubility among polymorphs have been reported to be typically less than 2.0fold. Although the utilization of metastable polymorphs is one of the effective approaches to enhance the dissolution rate of a drug, the metastable forms eventually transform to the thermodynamically stable form. It is necessary to monitor the polymorphic transformation during both manufacturing and storage of dosage forms to ensure reproducible bioavailability after oral administration (Zhang et al 2004).

\section{Salt formation}

In the pharmaceutical industry, salt formation approach is commonly used for an ionizable drug to increase solubility and dissolution rate. Salts are formed via proton transfer from an acid to a base. The counter ion containing salt changes the $\mathrm{pH}$ at the dissolving surface of a salt particle in the diffusion layer, resulting in a higher dissolution rate of the salts compared with that of the corresponding free forms. According to the Henderson-Hasselbalch equations, the change of $\mathrm{pH}$ highly influences the aqueous solubility of an ionizable drug (Avdeef, 2007). In theory, the solubility of a weak basic drug increases exponentially with decreasing $\mathrm{pH}$ at the $\mathrm{pH}$ range between its $\mathrm{pKa}$ and $\mathrm{pH} \max (\mathrm{pH}$ of maximum solubility in the pH-solubility profile). The increased saturation solubility on the dissolving surface contributes to the higher dissolution rate by salt formation. Celecoxib, a poorly water soluble weak acidic drug, showed an enhanced dissolution rate and oral bioavailability with a combination of $\mathrm{Na}$ salt formation and the use of a precipitation inhibitor compared with the corresponding free acid form. The solubility and dissolution rate of salt are influenced by the counter ion containing the salt. The solubility of haloperidol mesylate was significantly higher than that of its hydrochloride salt at a lower $\mathrm{pH}$ range. The aqueous solubility of a moderately soluble hydrochloride salt for a basic drug is sometimes reduced in solution containing chloride ion, such as gastric fluids (common-ion effects). An appropriate salt form should be developed from the viewpoints of both physicochemical and biopharmaceutical properties, especially for poorly water-soluble drugs.

\section{Cocrystal formation}

In recent years, much attention has been drawn to cocrystal for improving the dissolution rate of poorly water-soluble drugs. Cocrystal is broadly defined as crystalline materials comprised of at least two different components. Pharmaceutical cocrystal is typically composed of an API and a nontoxic guest molecule (cocrystal former) in a stoichiometric ratio. Unlike salt formation, 
proton transfer between the API and cocrystal former does not take place in cocrystal formation. In many cases, the API and cocrystal former require hydrogen bonding to form a stable cocrystal. Generally, $\mathrm{pKa}$ is one of the reliable indicators for distinguishing between salts and cocrystals, and the molecular complexes can be defined as a cocrystal when the pKa is less than 0 . When the pKa is between 0 and 3 , they can be salts or cocrystals or can contain sheared protons or mixed ionization states that cannot be assigned to either category. There have been several studies demonstrating the enhanced dissolution rate and oral bioavailability by cocrystal formation (Jung et al 2010).

\section{Particle size reduction \\ Micronization}

Particle size reduction approach is widely used to increase dissolution rate which proportionally increases with increasing surface area of drug particles. According to the Prandtl boundary layer equation, the decrease of diffusion layer thickness by reducing particle size, particularly down to $<5 \mu \mathrm{m}$, would result in accelerated dissolution. Thus, the increased surface area and the decreased diffusion layer thickness would lead to an enhanced dissolution rate of the drug. The common method to obtain micronized drug particles is mechanical pulverization of larger drug particles. Jet milling, ball milling, and pin milling are commonly used for dry milling. For solid powders, the lowest particle size that can be achieved by conventional milling is about 2-3 $\mu \mathrm{m}$. However, the milling does not always result in significantly enhancing the dissolution rate of the drug. Micronization sometimes increases agglomeration of the drug particles, which may decrease the surface area available for the dissolution. In such case, wetting agents, such as a surfactant, would play a major role in increasing the effective surface area. Micronization approach successfully enhanced the bioavailability of poorly water-soluble drugs such as griseofulvin, digoxin, and felodipine (Jounela et al 1975).

\section{Nanonization}

Particle size reduction to nano-meter range $(<1$ $\mu \mathrm{m})$ is an attractive and modern approach for poorly water-soluble drugs. Particle size reduction could lead to an increase of the surface area and a decrease of the diffusion layer thickness, which could provide an enhanced dissolution rate for drugs as well as an increase in the saturation solubility is also expected by reducing the particle size to less than $1 \mu \mathrm{m}$, as described by Ostwald-Freundlich's equation. The nanocrystal formulations are commonly produced by wet-milling with beads, highpressure homogenization, or controlled precipitation. Hydrophilic polymer and/or surfactant are typically used to stabilize nanocrystal suspension. The nanocrystalline drug particles are dispersed into inert carriers after a drying process, such as spray drying or lyophilization. Herein, the solidified nanocrystal formulations can be defined as crystalline solid dispersion (CSD). There have been numerous studies demonstrating the enhanced oral bioavailability of pharmaceuticals and neutraceuticals by nanocrystal and other technologies (Fakes et al 2009; Kawabata et al 2010; Chouksey et al 2011; Talegaonkar et al 2011; Mishra et al 2013).

\section{Amorphization}

Amorphous solids have higher energy than crystalline solids. Typically, the solubility of an amorphous drug is higher than that of the corresponding crystalline drug. The differences of the solubility between amorphous form and crystalline form have been reported to be between 1.1 and 1000-fold. The marked enhancement in the saturation solubility of amorphous drug may lead to a significant improvement of oral bioavailability. Stable amorphous formulations can be obtained by solid dispersion techniques. Amorphous solid dispersion (ASD) is defined as a distribution of active ingredients in molecular and amorphous forms surrounded by inert carriers (Chiou and Riegelman, 1971). The ASD formulations can be prepared by spray drying, melt extrusion, lyophilization, and use of supercritical fluids with polymeric carriers and/or surfactant. Numerous studies have demonstrated the marked enhancement of oral absorption (parameters such as Cmax and AUC) by ASD approaches as compared crystalline formulation containing bulk API or a physical mixture of API and carriers (Chen et al 2004; Sachan and Pushkar, 2011). However, ASD formulations tend to be chemically and physically less stable than the corresponding crystalline solid. The transformation from amorphous form to crystalline form in ASD formulation would lead to a reduction of oral bioavailability of the incorporated drugs. In contrast to the CSD 
formulations, the ASD approaches might be unsuitable for amorphous drugs with low stability.

\section{Cyclodextrin complexation}

Cyclodextrins are oligosaccharides containing a relatively hydrophobic central cavity and hydrophilic outer surface. Cyclodextrins have been widely used in pharmaceutical product development, and there are currently more than 10 marketed cyclodextrin-containing solid dosage forms. Cyclodextrins and their derivatives increase the apparent solubility of poorly water-soluble drugs by forming inclusion complexes. Numerous studies have demonstrated the enhancement of the oral bioavailability of poorly water-soluble drugs by the cyclodextrin inclusion complex (Rajewski and Stella, 1996; Brewster and Loftsson, 2007; Pabreja and Dua, 2011; Dahiya and Tayde, 2013).

\section{Self-emulsification}

In recent years, self-emulsification drug delivery systems (SEDDS) have been utilized to enhance the oral bioavailability of poorly water-soluble drugs, especially for highly lipophilic drugs. Selfemulsification formulations are isotropic mixtures of oil, surfactant, cosolvent, and solubilized drug (Gursoy and Benita, 2004). These formulations can rapidly form oil in water $(w / o)$ fine emulsions when dispersed in aqueous phase under mild agitation. SEDDS are additionally classified into selfmicroemulsification drug delivery systems (SMEDDS) and self-nanoemulsification drug delivery systems (SNEDDS) according to the size range of their oil droplets (Kohli et al 2010). SMEDDS form microemulsions ranging in droplet size from 100 to $250 \mathrm{~nm}$. Finer microemulsions of less than $100 \mathrm{~nm}$ can be obtained using SNEDDS. The rapid emulsification of these formulations in the gastrointestinal tract can provide both improved oral bioavailability and a reproducible plasma

\section{REFERENCES}

Amidon GL, Lennernas H, Shah VP, Crison JR. A theoretical basis for a biopharmaceutic drug classification: the correlation of in vitro drug product dissolution and in vivo bioavailability. Pharm. Res. 1995;12(3):413-20. [DOI: 10.1023/A:1016212804288]

Avdeef A. Solubility of sparingly-soluble ionizable drugs. Adv. Drug Deliv. Rev. 2007;59(7):568-90. [DOI: 10.1016/j. addr.2007.05.008]

Brewster ME, Loftsson T. Cyclodextrins as pharmaceutical solubilizers. Adv. Drug Del. Rev. 2007;59(7):645-66. concentration profile. The droplet size of the emulsion would influence the extent of absorption of the orally administered drugs.

\section{pH modification}

$\mathrm{pH}$ modification in solid dosage forms is considered to be an alternative option for an ionizable drug to improve the solubility and dissolution rate. The $\mathrm{pH}$ change significantly influences the saturation solubility of an ionizable drug by dissociation as the incorporation of $\mathrm{pH}$ modifiers in the dosage form can alter the microenvironmental $\mathrm{pH}$. Microenvironment is a term used to represent a microscopic layer surrounding a solid particle in which the solid forms a saturated solution of adsorbed water. The microenvironmental $\mathrm{pH}$ would affect the performance of the solid dosage form, such as the chemical stability of the drug substance and the dissolution profile. There have been several studies demonstrating the $\mathrm{pH}$ independent release of basic drugs form controlled release dosage forms by using $\mathrm{pH}$ modification technologies (Kranz et al 2005; Tatavarti and Hoag, 2006).

\section{CONCLUSION}

Solubility enhancement of poorly water soluble drugs have always been a challenge for formulation scientists, as solubility is the key determinant for drugs whose absorption is dissolution rate limited. The most fundamental approach so far is the particle size reduction, and various techniques have been attempted to attain ultimately, the desired in vivo performance once any approach is employed for the formulation development.

Since, many limitations are associated with the conventional particle size reduction technologies, continuous research is going on in search of better techniques over existing ones. To employ any of such technique, we need to consider various issues and concerns associated with these techniques and choose wisely among these.

Chen Y, Zhang GGZ, Neilly J, Marsh K, Mawhinney D, Sanzgiri YD. Enhancing the bioavailability of ABT-963 using solid dispersion containing Pluronic F-68. Int. J. Pharm. 2004;286(1-2):69-80. [DOI: 10.1016/j.ijpharm.20 04.08.009]

Chouksey R, Jain AK, Pandey $\mathrm{H}$, Maithil A. In vivo assessment of atorvastatin nanoemulsion formulation. Bull. Pharm. Res. 2011;1(2):10-4.

Dahan A, Miller JM, Amidon GL. Prediction of solubility and permeability class membership: provisional BCS 
classification of the world's top oral drugs. AAPS J. 2009;11(4):740-6. [DOI: 10.1208/s12248-009-9144-x]

Dahiya S, Tayde P. Binary and ternary solid systems of carvedilol with 2-hydroxypropyl- $\beta$-cyclodextrin and PVP K30. Bull. Pharm. Res. 2013;3(3):128-34.

Fakes MG, Vakkalagadda BJ, Qian F, Desikan S, Gandhi RB, Lai C, Hsieh A, Franchini MK, Toale H, Brown J. Enhancement of oral bioavailability of an HIV-attachment inhibitor by nanosizing and amorphous formulation approaches. Int. J. Pharm. 2009;370(1-2):167-74. [DOI: 10.1016/j.ijpharm.2008.11.018]

Galia E, Nicolaides E, Horter D, Löbenberg R, Reppas C, Dressman JB. Evaluation of various dissolution media for predicting in vivo performance of class I and II drugs. Pharm. Res. 1998;15(5):698-705. [DOI: 10.1023/A:10119 10801212]

Gursoy RN, Benita S (2004) Self-emulsifying drug delivery systems (SEDDS) for improved oral delivery of lipophilic drugs. Biomed. Pharmacother. 2004;58(3):173-82. [DOI: 10.1016/j.biopha.2004.02.001]

Hancock BC, Zografi G. Characteristics and significance of the amorphous state in pharmaceutical systems. J. Pharm. Sci. 1997;86(1):1-12. [DOI: 10.1021/js9601896]

Henck J-O, Griesser UJ, Burger A. Polymorphie von Arzneistoffen: Eine wirtschaftliche Herausforderung?. Pharm. Ind. 1997;59(2):165-9.

Horter D, Dressman JB. Influence of physicochemical properties on dissolution of drugs in the gastrointestinal tract. Adv. Drug Deliv. Rev. 2001;46(1-3):75-87. [DOI:10.1016/S0169-409X(00)00130-7]

Jounela AJ, Pentikainen PJ, Sothmann A. Effect of particle size on the bioavailability of digoxin. Eur. J. Clin. Pharmacol. 1975;8(5):365-70.

Jung MS, Kim JS, Kim MS, Alhalaweh A, Cho W, Hwang SJ, Velaga SP. Bioavailability of indomethacin-saccharin cocrystals. J. Pharm. Pharmacol. 2010;62(11):1560-8. [DOI: 10.1111/j.2042-7158.2010.01189.x]

Kawabata Y, Yamamoto K, Debari K, Onoue S, Yamada S. Novel crystalline solid dispersion of tranilast with high photostability and improved oral bioavailability. Eur. J. Pharm. Sci. 2010;39(4):256-62. [DOI: 10.1016/j.ejps.200 9.12.009]

Kohli K, Chopra S, Dhar D, Arora S, Khar RK. Selfemulsifying drug delivery systems: an approach to enhance oral bioavailability. Drug Discov. Today 2010;15(21-22):958-65. [DOI: 10.1016/j.drudis.2010.08. 007]

Kranz H, Guthmann C, Wagner T, Lipp R, Reinhard J. Development of a single unit extended release formulation for ZK 811 752, a weakly basic drug. Eur. J.
Pharm. Sci. 2005;26(1):47-53. [DOI: 10.1016/j.ejps.2005. 04.018]

Loftsson T, Brewster ME. Pharmaceutical applications of cyclodextrins. 1. Drug solubilization and stabilization. J. Pharm. Sci. 1996;85(10):1017-25. [DOI: 10.1021/js9505 34b]

Mishra DK, Kumar A, Raj R, Chaturvedi A. Capmul MCM based nanoemulsion for intranasal delivery of an antidepressant. Bull. Pharm. Res. 2013;3(1):34-9.

Noyes AA, Whitney WR. The rate of solution of solid substances in their own solutions. J. Am. Chem. Soc. 1897;19(12):930-4. [DOI: 10.1021/ja02086a003]

Pabreja K, Dua K. Comparative evaluation of in situ intestinal absorption of aceclofenac from solid dispersions, $\beta$-cyclodextrin complexes and co-precipitates in rats. Bull. Pharm. Res. 2011;1(1):26-30.

Rajewski RA, Stella VJ. Pharmaceutical applications of cyclodextrins. 2. In vivo drug delivery. J. Pharm. Sci. 1996;85(11):1142-69. [DOI: 10.1021/js960075u]

Sachan NK, Pushkar S. Solid dispersions: An industrially feasible alternative approach to formulate brick dust molecules. Bull. Pharm. Res. 2011;1(1):75-87.

Takagi T, Ramachandran C, Bermejo M, Yamashita S, Yu LX, Amidon GL. A provisional biopharmaceutical classification of the top 200 oral drug products in the United States, Great Britain, Spain, and Japan. Mol. Pharm. 2006;3(6):631-43. [DOI: 10.1021/mp0600182]

Talegaonkar S, Tariq M, Alabood RM. Design and development of $o / w$ nanoemulsion for the transdermal delivery of ondansetron. Bull. Pharm. Res. 2011;1(3): 18-30.

Tatavarti AS, Hoag SW. Microenvironmental pH modulation based release enhancement of a weakly basic drug from hydrophilic matrices. J. Pharm. Sci. 2006;95(7):1459-68. [DOI: 10.1002/jps.20612]

Wu C-Y, Benet LZ. Predicting drug disposition via application of BCS: transport/absorption/elimination interplay and development of a biopharmaceutics drug disposition classification system. Pharm. Res. 2005;22(1):11-23. [DOI: 10.1007/s11095-004-9004-4]

Yu LX, Amidon GL, Polli JE, Zhao H, Mehta MU, Conner DP, Shah VP, Lesko LJ, Chen ML, Lee VH, Hussain AS. Biopharmaceutics classification system: the scientific basis for biowaiver extensions. Pharm. Res. 2002;19(7): 921-5. [DOI: 10.1023/A:1016473601633]

Zhang GGZ, Law D, Schmitt EA, Qiu Y. Phase transformation considerations during process development and manufacture of solid oral dosage forms. Adv. Drug Deliv. Rev. 2004;56(3):371-90. [DOI: 10.1016/j.addr.2003.10.0 09] 